\title{
Remote Synchronization Experiments for Quasi-Zenith Satellite System Using Multiple Navigation Signals as Feedback Control
}

\author{
Toshiaki Iwata, ${ }^{1}$ Takashi Matsuzawa, ${ }^{2}$ Kumiko Machita, ${ }^{2}$ and Akiyoshi Abei ${ }^{3}$ \\ ${ }^{1}$ Collaborative Research Team for Verification, Kansai Collaboration Center, National Institute of Advanced Industrial Science and \\ Technology (AIST), 1-8-31 Midorigaoka, Ikeda, Osaka 563-8577, Japan \\ ${ }^{2}$ Space Technology Group, Energy Technology Research Institute, National Institute of Advanced Industrial Science and \\ Technology (AIST), 1-1-1 Umezono, Central 2, Tsukuba, Ibaraki 305-8568, Japan \\ ${ }^{3}$ Cosmo Research Corporation, 3-148-5 Miyahara-cho, Kita-ku, Saitama, Saitama 331-0812, Japan
}

Correspondence should be addressed to Toshiaki Iwata, totty.iwata@aist.go.jp

Received 30 July 2010; Revised 19 December 2010; Accepted 15 March 2011

Academic Editor: Patrizia Tavella

Copyright (c) 2011 Toshiaki Iwata et al. This is an open access article distributed under the Creative Commons Attribution License, which permits unrestricted use, distribution, and reproduction in any medium, provided the original work is properly cited.

\begin{abstract}
The remote synchronization system for the onboard crystal oscillator (RESSOX) is a remote control method that permits synchronization between a ground station atomic clock and Japanese quasi-zenith satellite system (QZSS) crystal oscillators. To realize the RESSOX of the QZSS, the utilization of navigation signals of QZSS for feedback control is an important issue. Since QZSS transmits seven navigation signals (L1C/A, L1CP, L1CD, L2CM, L2CL, L5Q, and L5I), all combinations of these signals should be evaluated. First, the RESSOX algorithm will be introduced. Next, experimental performance will be demonstrated. If only a single signal is available, ionospheric delay should be input from external measurements. If multiple frequency signals are available, any combination, except for L2 and L5, gives good performance with synchronization error being within two nanoseconds that of RESSOX. The combination of L1CD and L5Q gives the best synchronization performance (synchronization error within $1.14 \mathrm{~ns}$ ). Finally, in the discussion, comparisons of long-duration performance, computer simulation, and sampling number used in feedback control are considered. Although experimental results do not correspond to the simulation results, the tendencies are similar. For the overlapping Allan deviation of long duration, the stability of $1.23 \times 10^{-14}$ at $100,160 \mathrm{~s}$ is obtained.
\end{abstract}

\section{Introduction}

The Japanese Quasi-Zenith Satellite System (QZSS) is a three-satellite navigation/positioning system conceived to improve the positioning performance (satellite availability and position accuracy) of the presently available global positioning system (GPS) for civilians in urban areas where high-rise buildings exist and where mountains reduce the number of visible GPS satellites in Japan [1]. The orbits are in inclined orbital planes (43 degrees) from the equatorial plane, although the semimajor axis is the same as that of geostationary satellites.

In a navigation satellite system, the onboard clock system is of primary importance because the position is calculated on the basis of the signal transmitting time from the satellite to the point, where the signal transmission velocity is 299 ,
$792,458 \mathrm{~m} / \mathrm{s}$. If the time system has a $10 \mathrm{~ns}$ error, a $3 \mathrm{~m}$ pseudorange error will arise.

The proposed Japanese QZSS has the following properties regarding its timekeeping system (TKS): (1) it is possible to control the system over a 24 -hour period as long as a good ground site is available, such as Okinawa, Japan, (2) a high-stability crystal oscillator is superior to an atomic clock in terms of short-term frequency stability [2], (3) ground stations are continuously operational, and (4) QZSS employs a maximum of three satellites, which is not too many to monitor from the ground.

The remote synchronization system for an onboard crystal oscillator (RESSOX) has been planned by the National Institute of Advanced Industrial Science and Technology (AIST) since 2003 [3]. RESSOX is a remote control method that permits synchronization between a ground station 
atomic clock and QZSS crystal oscillators. The targets of RESSOX are synchronization within $10 \mathrm{~ns}$ between the ground station time standard and the onboard QZSS crystal oscillators and stability better than $1 \times 10^{-13}$ at $100,000 \mathrm{~s}$.

RESSOX reduces the overall cost, satellite power consumption, and onboard weight and volume and has a longer lifetime than a system with onboard atomic clocks. However, we should note that QZSS will carry two rubidium atomic clocks, and RESSOX is to be used in the experiments because of their intended use in QZSS in the future.

Similar techniques in which onboard atomic clocks are not used have been proposed. For example, Kawase proposed a satellite communication-navigation system that transmits a navigation signal from a ground station to the communication satellite and sends it back to the Earth immediately [4]. In this case, obstacles, such as airplanes or low-Earthorbit satellites encountered during uplink transmission, and interruption of the uplink to avoid interference with geostationary communication satellites produce fatal effects. The science satellite "HALCA" uses the phase transfer system in space for very long baseline interferometry (VLBI) observations under the VLBI Space Observatory Program [5]. In this method, only phase information, not time information, is used to adjust the frequency at the ground station, and a $65.36 \mathrm{ps}$ ambiguity (carrier frequency is $15.3 \mathrm{GHz}$ ) occurs if time extraction is attempted. Another similar research on a time and frequency dissemination system using $\mathrm{GEO} / \mathrm{MEO} / \mathrm{LEO}$ satellites and TDMA was proposed by Giunta et al. [6].

\section{RESSOX Overview}

Figure 1 shows the schematic of RESSOX. In order to realize RESSOX, it is essential to identify the delay/error factors and the feedback mechanism by measuring the delay at the ground station. The former is related to the estimation of error and delay using models, and is considered to be a feedforward loop. The RESSOX control signal includes the time information of the ground atomic clock and is advanced to compensate the transmission delay. Thus, the RESSOX control signal is synchronized with the ground atomic clock when it arrives at the QZSS. The onboard crystal oscillator is controlled to follow the RESSOX control signal.

Navigation signals are broadcast from the QZSS on the basis of the crystal oscillator time. On the ground, the QZSS/GPS receiver measures the pseudoranges of the navigation signals. The difference between measured and estimated pseudoranges is used to adjust the RESSOX control signal. This is considered as feedback control.

The error and delay models in the feed-forward loop are for delays in the ground station and in the satellite, tropospheric delay, ionospheric delay, delay due to distance (orbit estimation), delay due to relativity effects, and errors caused by Earth's motion, such as daily rotation, nutation, and precession. These problems were discussed in our previous paper [7]. However, if multiple navigation signals are used for feedback, the use of the delay models of the troposphere and ionosphere becomes unnecessary $[8,9]$.

\section{Experimental Apparatuses}

Since each apparatus has been introduced in detail in [10], we here explain them only briefly. The block diagram of the experimental setup is shown in Figure 2.

A RESSOX control signal transmitter (RCST) that advances the time information to compensate the transmission delay, a QZSS/GPS receiver (QZSSREC) that measures the pseudoranges of the navigation signals, the RESSOX controller (RC) that comprises a PC using Windows XP, and a frequency transformer (FT) that generates $10.23 \mathrm{MHz}$ and $1.5 \mathrm{~s}$ pulses were provided as ground station equipment. As the reference clock, a hydrogen maser (H-Maser) is used. An uplink delay simulator (UDS2), an engineering model of the onboard crystal oscillator (MINI-OCXO), a simulator of the onboard time comparison unit (TCUSIM), a simulator of the navigation onboard computer (NOCSIM), a D/A converter, a QZSS simulator (QSIM2) that provides navigation signals with transmission delay, and a pulse generator (PG) of $1 \mathrm{~s}$ and $1.5 \mathrm{~s}$ pulses were also provided to confirm the operation of the ground station apparatuses. A time interval counter (TIC) measures the time difference between $\mathrm{H}$-Maser and MINI-OCXO. Allan deviation of MINI-OCXO during free running (constant control voltage) is shown in Figure 3.

\section{Control Methods}

The RESSOX control algorithm is outlined as follows (please refer to Figures 2 and 4 and Table 1).

Step 1 (Model Delay Preparation (RDP)). Four (L1/L2/L5/ Ku-band signals) model delay files that do not contain any errors are prepared. We assume that all models (orbit, ionosphere, troposphere, etc.; see Table 1) are correct. Three (L1/L2/L5-band navigation signals) delay files include the times (date and UTC) at which the L1/L2/L5-band navigation signals are received at the ground station, and the model delays of the L1/L2/L5-band navigation signals. On the basis of the model delays, a scenario data file in the CSV format for SimQZ that controls QSIM2 is generated. Another delay file that is used as the Ku delay file for UDS2 includes the time (date and UTC) at which the Ku-band signal is transmitted from the ground station.

Step 2 (Delay Estimation with Error (DE)). We prepare four (L1/L2/L5/Ku-band signals) estimated delay files calculated using models such as those of delays due to the orbit, ionosphere, or troposphere with random and/or systematic errors, and we assume that their parameters are obtained at the ground station as measurement results. Five-meter errors of each coordinate in the International Celestial Reference Frame (ICRF) are assumed for the initial satellite position. Three (L1/L2/L5-band navigation signals) delay files include the times (date and UTC) at which the L1/L2/L5-band navigation signals are received at the ground station and the estimated delays of the L1/L2/L5-band navigation signals. Another delay that is contained in the Ku-band estimated time adjustment file includes the time (date and UTC) at which the Ku-band signal is transmitted from the ground 


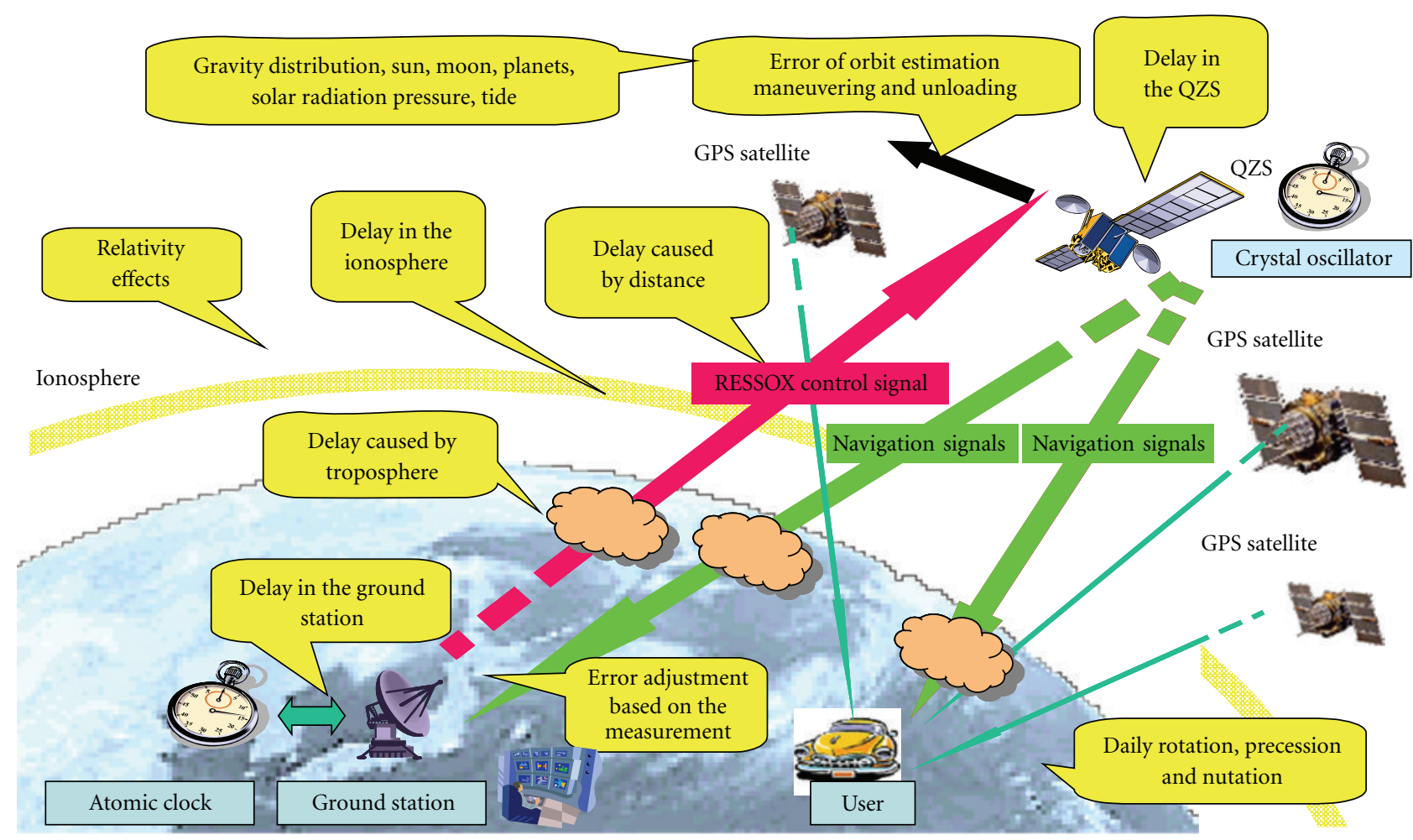

FIGURE 1: RESSOX schematic.

station and the estimated delay of the Ku-band signal. These estimated delays are converted into a database of L1/L2/L5-band delays and the time adjustment file for RCST, respectively. In this experiment, when multiple frequency navigation signals are used, ionospheric, tropospheric, and relativity effects are not estimated. However, when only a single-frequency navigation signal is used, we assume that these effects are estimated completely. The ionosphere map was provided by the Center for Orbit Determination in Europe (CODE), and tropospheric delay was calculated with the Saastamoinen model. Unfortunately, the above assumption is inappropriate because such an ionospheric model is not available in real time. We used this CODE map to enable a comparison with multiple frequency cases in which the ionospheric delay in the experiments can be estimated. Table 1 shows the assumptions made in the calculations in Steps 1 and 2.

Step 3 (RCST Operation with Feed-Forward Contol). The Ku-band time adjustment file for RCST is fed into the RCST as feed-forward control, and RCST advances the time information of the ground atomic clock as the RESSOX control signal.

Step 4 (UDS2 Operation). UDS2 delays the RESSOX control signal in accordance with the Ku-band delay file for UDS2.

Step 5 (MINI-OCXO Control). MINI-OCXO is controlled by NOCSIM and the D/A converter using the time difference between the RESSOX control signal at TCUSIM and the time of MINI-OCXO measured by TCUSIM.NOCSIM calculates the voltage using the following formula:

$$
\begin{aligned}
v_{k}= & v_{\text {offset }}-\frac{K_{1}}{l+1} \sum_{i=k-1}^{k}\left(t_{\text {OCXO }}-t_{\text {RESSOX }}\right)_{i} \\
& -K_{2} \sum_{i=0}^{k-p} \int_{i}^{i+p}\left(t_{\text {OCXO }}-t_{\text {RESSOX }}\right) d t,
\end{aligned}
$$

where $v_{k}$ is the $k$ th applied voltage, $v_{\text {offset }}=5.4(\mathrm{~V}), K_{1}$ is a proportional gain set at $7.0 \times 10^{5}(\mathrm{~V} / \mathrm{s}), K_{2}$ is an integral gain set at $3.0 \times 10^{3}\left(\mathrm{~V} / \mathrm{s}^{2}\right), l$ is the number of past data used for proportional control set at $1, k$ is the data number from the beginning, $p$ is the integral interval, which means an overlapping integral number, set at $3, t_{\mathrm{OCXO}}$ is the time of MINI-OCXO, and $t_{\text {RESSOX }}$ is the time of the RESSOX control signal. $K_{1}$ tries to reduce time error, while $K_{2}$ aims to reduce the drift.

Step 6 (QSIM2 Control). QSIM2 generates L1/L2/L5-band navigation signals according to the scenario data file in the CSV format for SimQZ.

The procedures from Step 7 to Step 9 make up feedback control (FB).

Step 7 (Error Estimation). Pseudoranges of L1/L2/L5-band navigation signals obtained by the QZSSREC are compared with the database of L1/L2/L5-band delays prepared in Step 2, and the differences between the pseudoranges and 


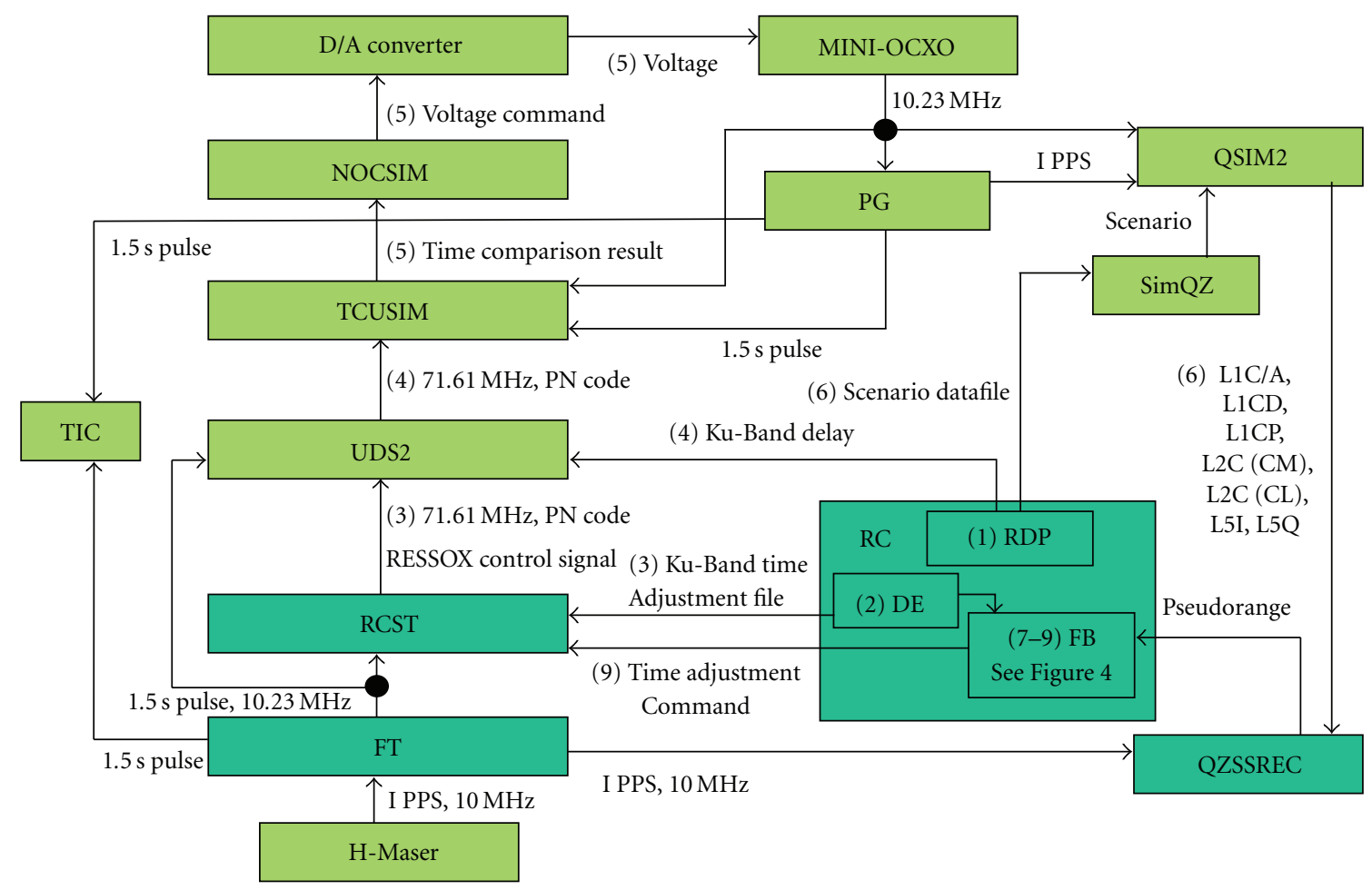

() indicates step number in Section 4

Apparatuses of ground station

Apparatuses for validation confirmation

FIGURE 2: Block diagram of experimental setup.

TABLE 1: Assumptions in calculations.

\begin{tabular}{|c|c|c|c|}
\hline Condition of orbit and delay calculation & No error (for UDS2 and QSIM2) & \multicolumn{2}{|c|}{ Error (for RCST and QZSSREC) } \\
\hline Navigation signals used for feedback & & Single frequency & Multiple frequency \\
\hline \multirow{6}{*}{ Initial condition of orbit calculation (ICRF) } & $x=-23342012.770 \mathrm{~m}$ & \multicolumn{2}{|c|}{$x=-23342007.770 \mathrm{~m}$} \\
\hline & $y=-33282936.570 \mathrm{~m}$ & \multicolumn{2}{|c|}{$y=-33282931.570 \mathrm{~m}$} \\
\hline & $z=15995302.769 \mathrm{~m}$ & \multicolumn{2}{|c|}{$z=15995307.7695 \mathrm{~m}$} \\
\hline & $v x=2184.551 \mathrm{~m} / \mathrm{s}$ & \multicolumn{2}{|c|}{$v x=2184.551 \mathrm{~m} / \mathrm{s}$} \\
\hline & $v y=-935.546 \mathrm{~m} / \mathrm{s}$ & \multicolumn{2}{|c|}{$v y=-935.546 \mathrm{~m} / \mathrm{s}$} \\
\hline & $v z=1774.093 \mathrm{~m} / \mathrm{s}$ & \multicolumn{2}{|l|}{$v z=1774.093 \mathrm{~m} / \mathrm{s}$} \\
\hline Gravity potential model & \multicolumn{3}{|c|}{ EGM96, 360 degree, 360 order } \\
\hline Other bodies & \multicolumn{3}{|c|}{ Moon, Sun, Mercury, Venus, Mars, Jupiter, Saturn,Uranus, Neptune, Pluto } \\
\hline Solar radiation pressure model & \multicolumn{3}{|c|}{ Cr model, $\mathrm{Cr}=1.2,30 \mathrm{~m}^{2}$} \\
\hline Solid tide effect & \multicolumn{3}{|c|}{ considered } \\
\hline Satellite mass & \multicolumn{3}{|c|}{$1816 \mathrm{Kg}$} \\
\hline Ionospheric delay & \multicolumn{2}{|c|}{ Code data are used } & Not considered \\
\hline Tropospheric delay & \multicolumn{2}{|c|}{ Saasamoinen, tempreature $15^{\circ} \mathrm{C}$, pressure $1013.25 \mathrm{hPa}$, humidity $70 \%$} & Not considered \\
\hline Relativity effect & \multicolumn{2}{|c|}{ considered } & Not considered \\
\hline Simulation period & \multicolumn{3}{|c|}{ 1999/12/31 23:59:47 - 2000/01/01 23:59:47 } \\
\hline Observed position & \multicolumn{3}{|c|}{ Okinawa $(26.5 \mathrm{~N}, 127.9 \mathrm{E}, 0.0 \mathrm{~m}$ geodetic height) } \\
\hline
\end{tabular}




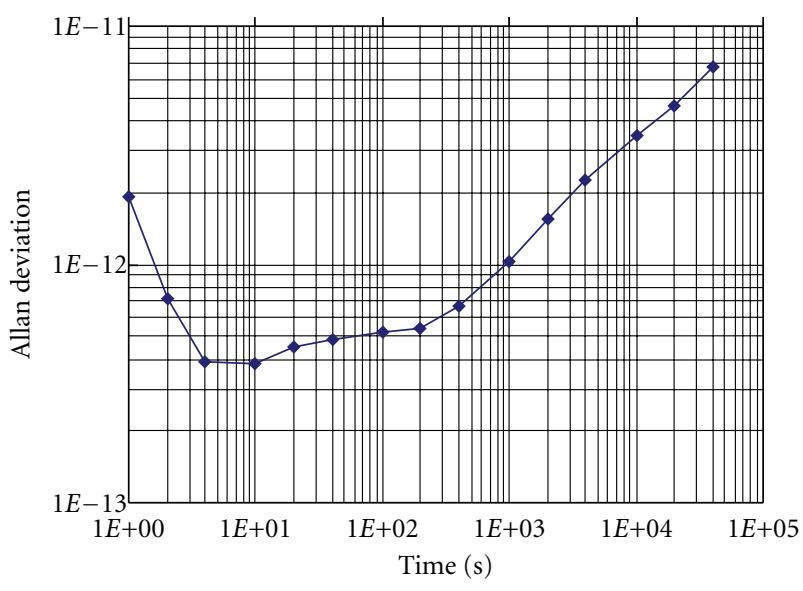

FIgure 3: Allan deviation of MINI-OCXO during free running.

the database are designated as $E_{1}$ for $\mathrm{L} 1$ (frequency $f_{\mathrm{L} 1}=$ $\left.1.57542 \times 10^{9} \mathrm{~Hz}\right), E_{2}$ for L2 $\left(f_{\mathrm{L} 2}=1.2276 \times 10^{9} \mathrm{~Hz}\right)$, and $E_{3}$ for $\mathrm{L} 5\left(f_{\mathrm{L} 5}=1.17645 \times 10^{9} \mathrm{~Hz}\right)$.

Step 8 (Separation of Ionospheric Error and Nonionospheric Error). The system of (2), (3), and (4), which includes $E_{1}, E_{2}$, and $E_{3}$ and delays due to the nonfrequency-dependent term $e$ and the coefficient of delay $k$ due to the frequency-dependent term (i.e., ionospheric delay) as unknowns, is prepared

$$
\begin{gathered}
e+\frac{k}{f_{\mathrm{L} 1}^{2}}=E_{1}, \quad f_{\mathrm{L} 1}=1.57542 \times 10^{9}(\mathrm{~Hz}), \\
e+\frac{k}{f_{\mathrm{L} 2}^{2}}=E_{2}, \quad f_{\mathrm{L} 2}=1.2276 \times 10^{9}(\mathrm{~Hz}), \\
e+\frac{k}{f_{\mathrm{L} 5}^{2}}=E_{3}, \quad f_{\mathrm{L} 5}=1.17645 \times 10^{9}(\mathrm{~Hz}) .
\end{gathered}
$$

If the navigation signals of all three frequencies are available, the system of (2), (3), and (4) is expressed as follows:

$$
\left[\begin{array}{cc}
1 & 1 / f_{L 1}^{2} \\
1 & 1 / f_{L 2}^{2} \\
1 & 1 / f_{L 5}^{2}
\end{array}\right]\left[\begin{array}{l}
e \\
k
\end{array}\right]=\mathbf{A x}=\left[\begin{array}{c}
E_{1} \\
E_{2} \\
E_{3}
\end{array}\right]=\mathbf{E} .
$$

This means that three equations exist for two unknown parameters ( $e$ and $k$ ). Therefore, the solution of simultaneous equations (1), (2), and (3) is given as follows:

$$
\mathbf{x}=\left(\mathbf{A}^{T} \mathbf{A}\right)^{-1} \mathbf{A}^{T} \mathbf{E}
$$

If the navigation signals of two frequencies are available, then two equations exist with two unknown parameters. Using the solutions of the system of equations, we obtain the time to be adjusted of the RESSOX control signal using the $\mathrm{Ku}$ band $\left(f_{\mathrm{Ku}}=1.43453 \times 10^{10} \mathrm{~Hz}\right)$ for the RCST

$$
e+\frac{k}{f_{\mathrm{Ku}}^{2}}, \quad f_{\mathrm{Ku}}=1.43453 \times 10^{10}(\mathrm{~Hz}) .
$$

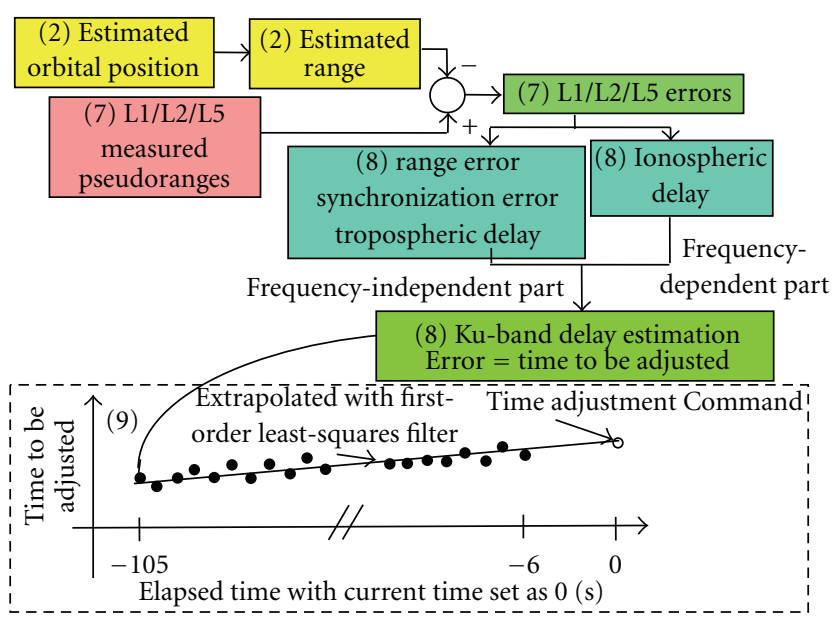

FIgURE 4: RESSOX algorithm used in Steps 7-9.

If the navigation signals of only one frequency are available, the time to be adjusted is given by $E_{i}(i=1$ or 2 or 3$)$.

Step 9 (Feedback Command Generation). By combining the time adjustment file in Step 2 and the time adjustment command based on the time to be adjusted in Step 8, RCST is controlled. We consider some filters in this step, as described later. Nominally, 100 values (i.e., $100 \mathrm{~s}$ ) of the time to be adjusted, which are found between before $6 \mathrm{~s}$ and before $105 \mathrm{~s}$, are extrapolated with the first-order least-squares filter and the time adjustment command is generated. Then, we go back to Step 4. The calculations of the time to be adjusted and the time adjustment command are conducted every second.Figur 4 shows the block diagram of the RESSOX algorithm using the three-frequency navigation signals in Steps 7 to 9 .

\section{Experiments}

5.1. Experimental Cases. The number of combinations of navigation signals using feedback control is 35 , as shown in Figure 5 and all combinations are examined for their effectiveness in feedback control. In the figure, light green indicates experiments using only one-frequency navigation signals (seven cases), pink the combinations of L1 and L2 (six cases), yellow the combinations of L1 and L5 (six cases), light blue the combinations of L2 and L5 (four cases), and light brown the combinations of L1, L2 and L5 (12 cases).

5.2. Experimental Results. In this section, representative experimental results of each combination are shown first, and evaluations of stability are indicated. Their stability performances are similar; they have a peak at approximately $100 \mathrm{~s}$, the origin of which is considered to be $100 \mathrm{~s}$ of the ground feedback time shown in Figure 4.

(1) Single-Frequency Cases. As a representative result, the case of L1CD (Ex. \#3) is shown in Figure 6. In this case, the 


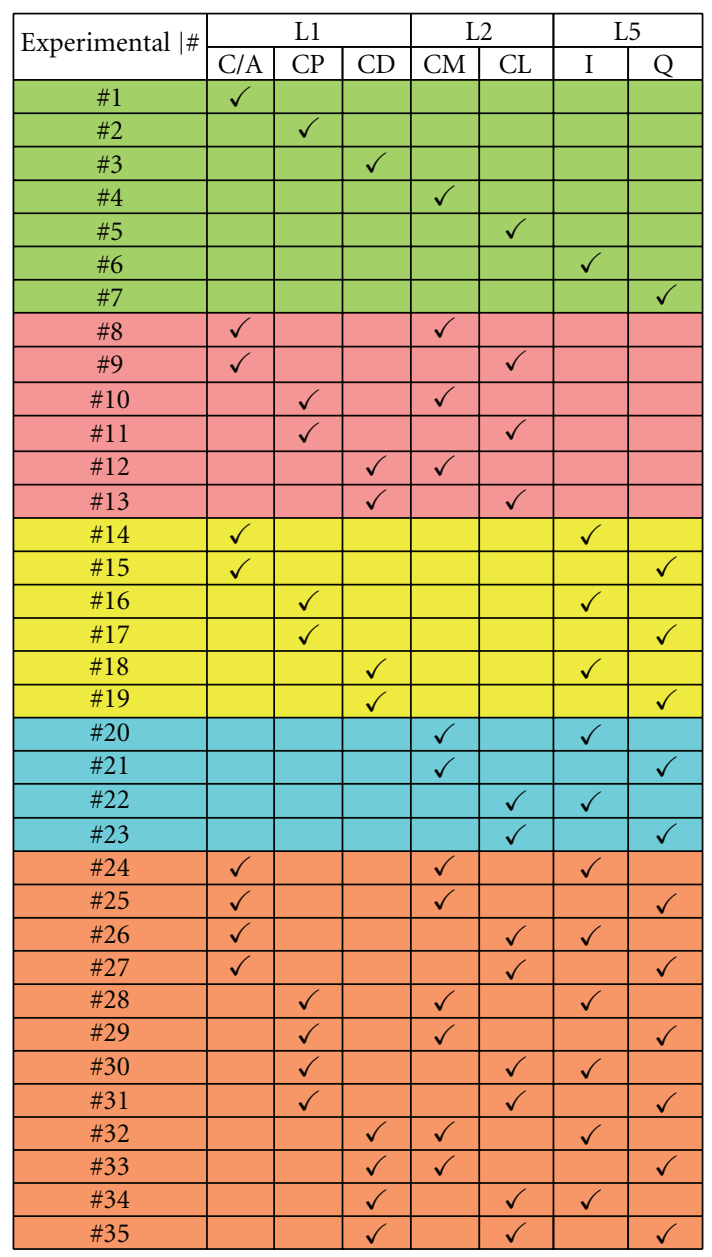

FIgURE 5: Experimental cases.

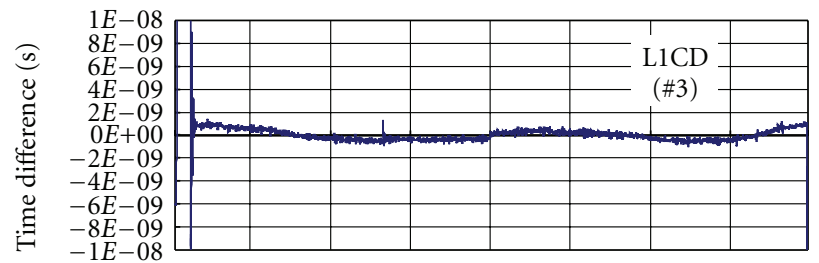

00/1/1 00/1/1 00/1/1 00/1/1 00/1/1 00/1/1 00/1/1 00/1/1 00/1/2

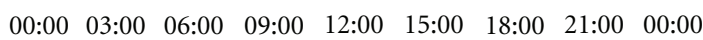
Date and time (UTC)

FIGURE 6: Representative result of single-frequency feedback.

synchronization error is within $2 \mathrm{~ns}$ throughout 24 hours. To compare the stability performance, Allan deviations of all seven cases are shown in Figure 7. Although the stabilities of all cases up to $10 \mathrm{~s}$ are almost the same, the stabilities of L5 signals are approximately one order better than those of other signals after $100 \mathrm{~s}$. The reason is that chip rates of L5 signals are 10 times faster than those of other signals and pseudorange deviations of L5 signals are approximately 10 times smaller than those of other signals.

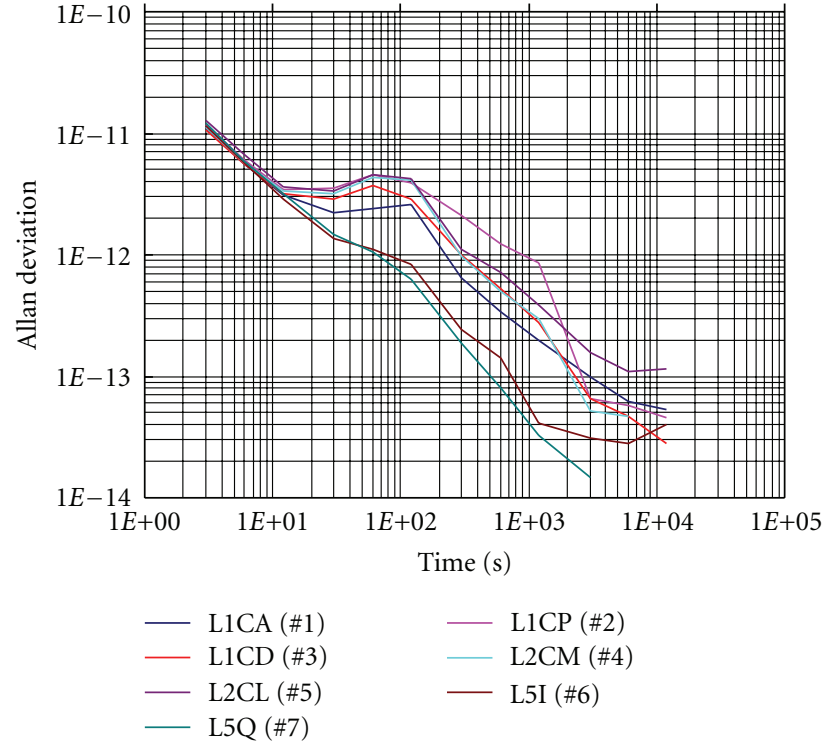

Figure 7: Allan deviations of single-frequency feedback.

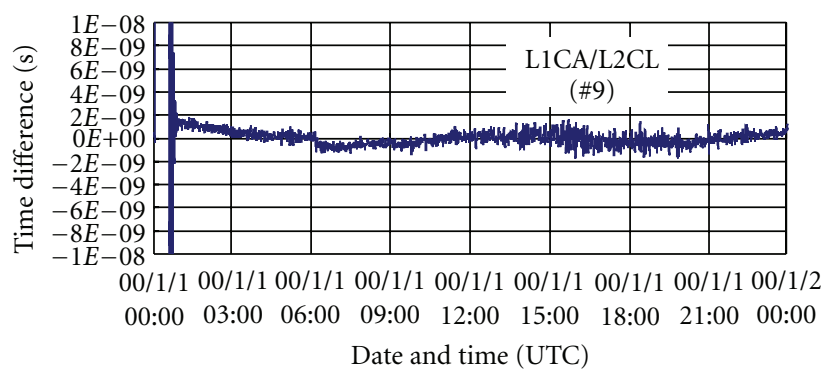

FIGURE 8: Representative result of L1/L2-frequency feedback.

(2) L1/L2 Combinations. As a representative result, the case of L1CA/L2CL (Ex. \#9) is shown in Figure 8. In this case, the synchronization error is also within $2 \mathrm{~ns}$ throughout 24 hours. To compare the stability performance, Allan deviations of all six cases are shown in Figure 9. In general, these results are worse than those of single-frequency cases.

(3) L1/L5 Combinations. As a representative result, the case of L1CP/L5Q (Ex. \#17) is shown in Figure 10. In this case, the synchronization error is also within 2 ns throughout 24 hours. To compare the stability performance, Allan deviations of all six cases are shown in Figure 11. Their stability performances are similar and peaks at approximately $100 \mathrm{~s}$ are smaller than those in L1/L2 cases. This means that L1/L 5 cases have better characteristics than L1/L2 cases. This is because L5 pseudoranges are more stable than that of L2, as shown in (1) of this section.

(4) L2/L5 Combinations. As a representative result, the case of $L 2 C L / L 5 I$ (Ex. \#22) is shown in Figure 12. In this case, the synchronization error is over $10 \mathrm{~ns}$ during 24 hours. The reason for this large error is that L2 and L5 frequencies are so close that the uncertainty in ionospheric delay estimation is 

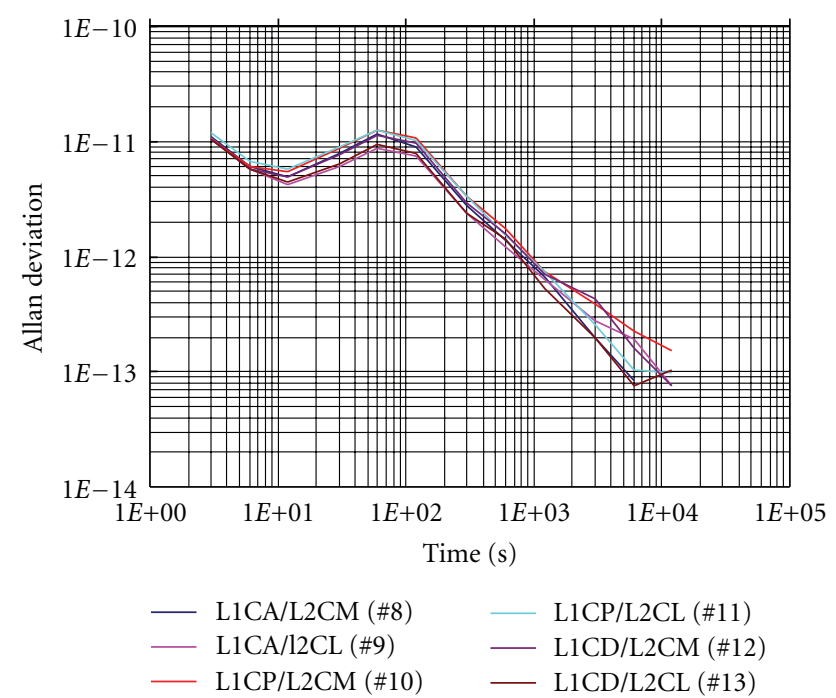

FIGURE 9: Allan deviations of L1/L2-frequency feedback.

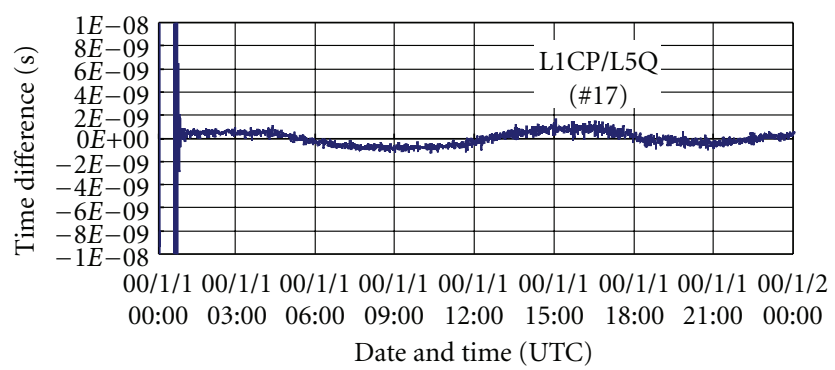

FIGURE 10: Representative result of L1/L5-frequency feedback.

large. To compare the stability performance, Allan deviations of four cases are shown in Figure 13. The stability of this group is one order worse than that of L1/L2, L1/L5 or L1/L2/L5 groups.

(5) L1/L2/L5 Combinations. As a representative result, the case of L1CA/L2CM/L5Q (Ex. \#25) is shown in Figure 14. In this case, the synchronization error is also within $2 \mathrm{~ns}$ throughout 24 hours. To compare the stability performance, Allan deviations of all 12 cases are shown in Figure 15. The characteristic of L1/L2/L5 combinations is similar to that of L1/L 5 cases.

\section{Discussion}

6.1. Long-Duration Stability. To confirm the performance of long-duration stability, the L1CA/L5I combination was selected and tested in a seven-day operation. The result of overlapping Allan deviation is shown in Figure 16. Even after ten thousand seconds, overlapping Allan deviation decreased and became $1.23 \times 10^{-14}$ at 100,160 s. Overlapping Allan deviation of the reference H-Maser, RH401A fabricated by ANRITSU, is also shown in Figure 15. From one to $1000 \mathrm{~s}$, overlapping Allan deviation of $\mathrm{H}$-Maser are two orders better than that of RESSOX using L1CA/L5I. This is because RESSOX is controlled on the basis of the results of a time

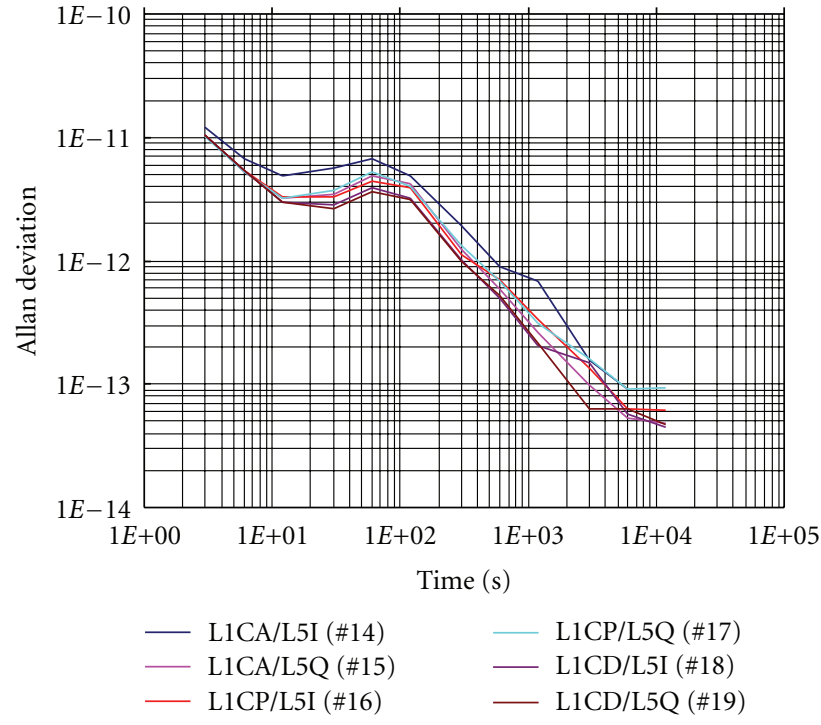

FIgURE 11: Allan deviations of L1/L5-frequency feedback.

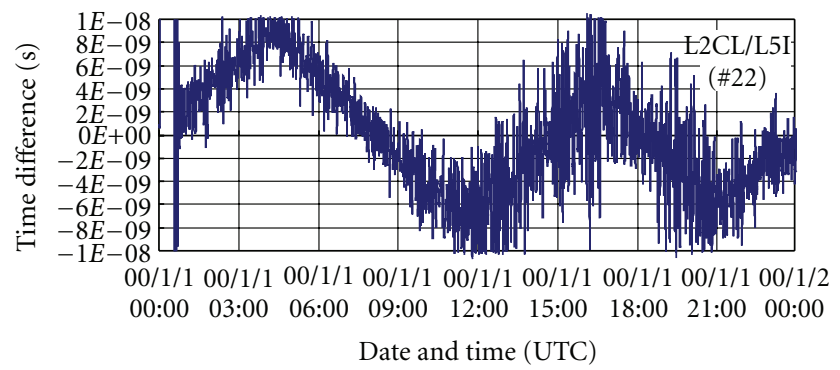

FIGURE 12: Representative result of L2/L5-frequency feedback.

comparison of TCUSIM, and because TCUSIM has $0.3 \mathrm{~ns}$ error in every second in the form of white phase noise. If we use not the PN code but the carrier phase of the RESSOX control signal, overlapping Allan deviation of RESSOX would be more greatly improved.

6.2. Comparison with Computer Simulation. Maximum synchronization errors of each combination have already been estimated by computer simulation using standard deviations of the pseudoranges of all signals measured with QZSSREC [9]. The results are shown in Figure 17. In the figure, cases without ionospheric delay (this means that ionospheric delay is completely estimated and included in feed-forward calculation; the conditions are the same as those in the experiments of the single-frequency case shown in Table 1) and the cases with ionospheric delay (this means that ionospheric delay is not estimated and not included in feedforward calculation; the conditions are the same as those in the experiments of the multiple frequency case shown in Table 1) are considered. In the case of single frequency, since it is difficult to estimate the ionospheric delay precisely, the synchronization error would be as large as that in the cases with ionospheric error of more than $10 \mathrm{~ns}$.

Using Allan deviations of each experimental result at $120 \mathrm{~s}$ the maximum synchronization errors of experimental 


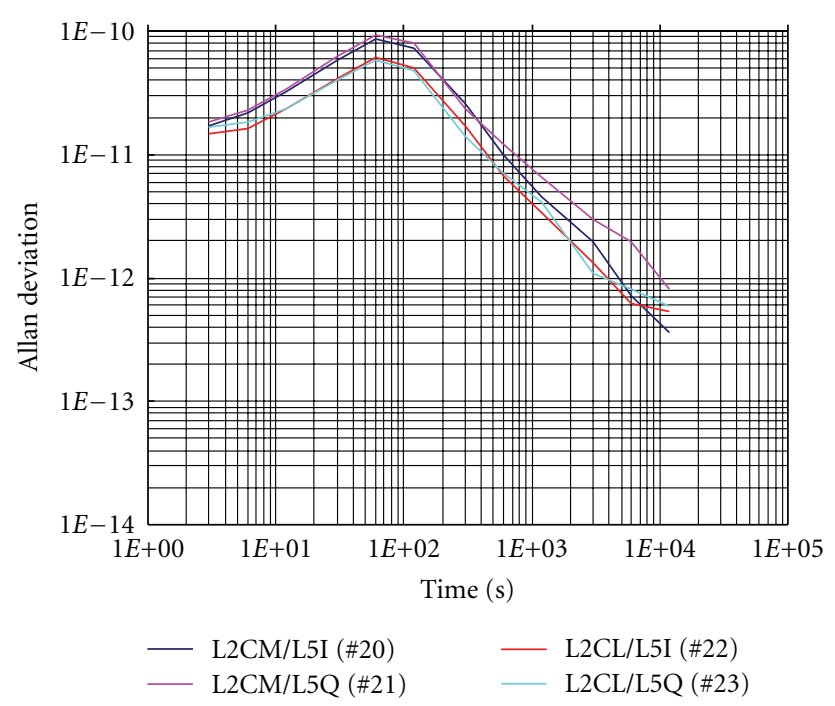

FIGURE 13: Allan deviations of L2/L5-frequency feedback.

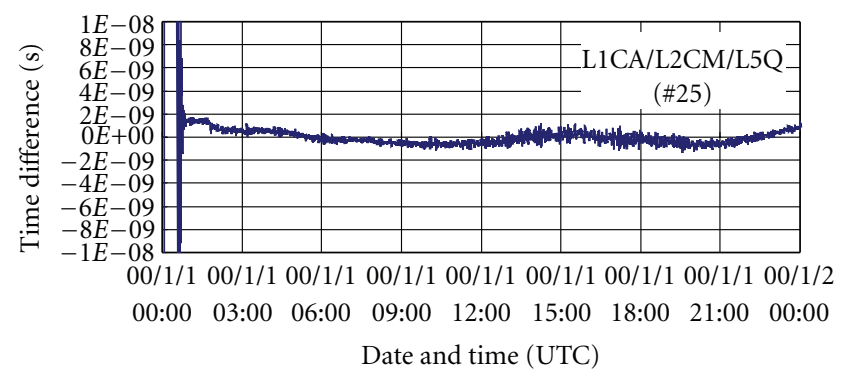

Figure 14: Representative result of L1/L2/L5-frequency feedback.

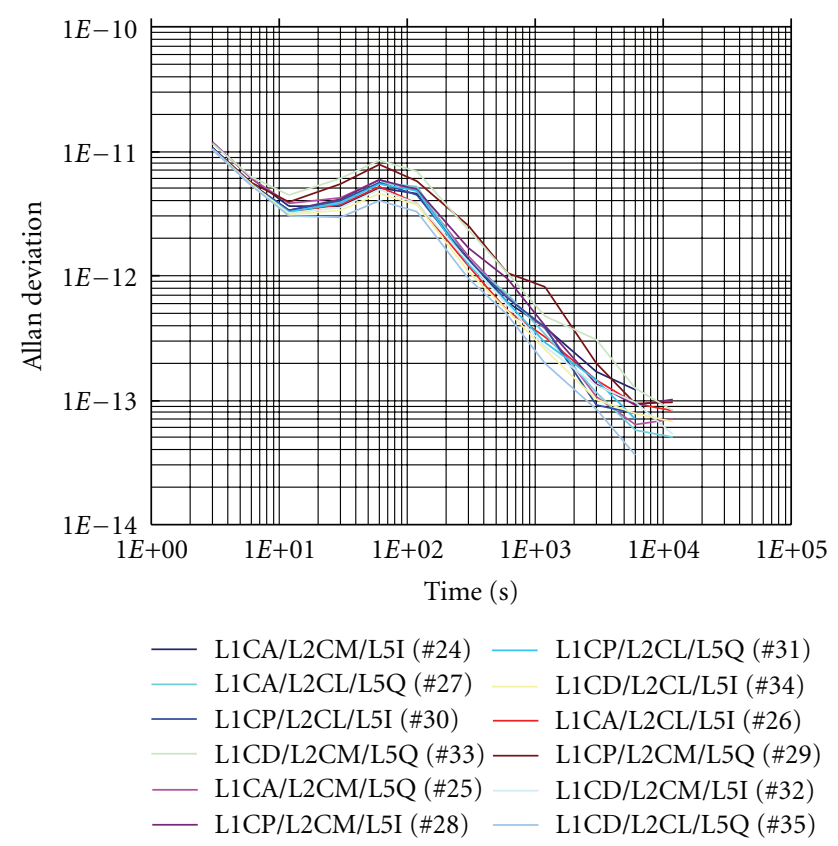

FIGURE 15: Allan deviations of L1/L2/L5-frequency feedback.

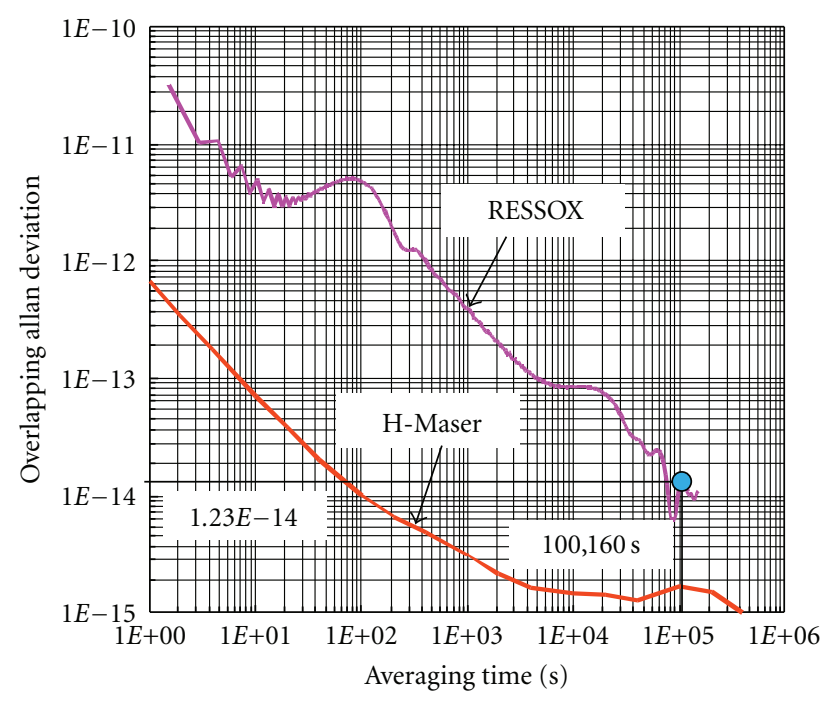

Figure 16: Overlapping Allan deviation of L1CA/L5I over a long duration.

results were estimated. In general, Allan deviation of experiments has a peak at $120 \mathrm{~s}$, and causes the worst error in the estimation. The maximum synchronization error $E_{\max }$ is estimated as

$$
E_{\max }=3 \times 120 \times \sigma_{120}
$$

where $\sigma_{120}$ is Allan deviation at $120 \mathrm{~s}$, and the range of $3 \sigma$ includes $99 \%$ statistical samples, so that this range can be considered the maximum range. The results are shown overlapped in Figure 17. The results do not agree with those of the simulation, however, the tendency of the experimental synchronization error is the same as that in the simulation. The combination yielding the best experimental results, excepting the single-frequency signal, is that of L1CD and L5Q, and the expected maximum synchronization error is $1.14 \mathrm{~ns}$ (in simulation, $3.4 \mathrm{~ns}$ ). The maximum synchronization error results of each combination can be used to assign the priority of navigation signal selection in RESSOX.

6.3. Effect of Number of Values of Time to Be Adjusted. To investigate the effect of the number of values of time to be adjusted in feedback on the ground shown in Figure 4, the combination of L1CA/L2CL/L5Q was selected and examined by experiment. The numbers of values of time to be adjusted were 50, 100, 200, and 500 (corresponding elapsed times for feedback control were 50,100, 200, and $500 \mathrm{~s}$ ). The results of Allan deviation are shown in Figure 18. As expected, the peaks corresponding to the number of values of time to be adjusted were observed. To improve the short-term stability, the number of values of time to be adjusted should be large, however, the time required to converge the synchronization (i.e., time constant) increases. To improve the stability of RESSOX control, one strategy would be to use 100 values of time to be adjusted (elapsed time for feedback control is $100 \mathrm{~s}$ ) in the convergence phase and 500 values (elapsed time for feedback control is $500 \mathrm{~s}$ ) in the steady phase in feedback control. 


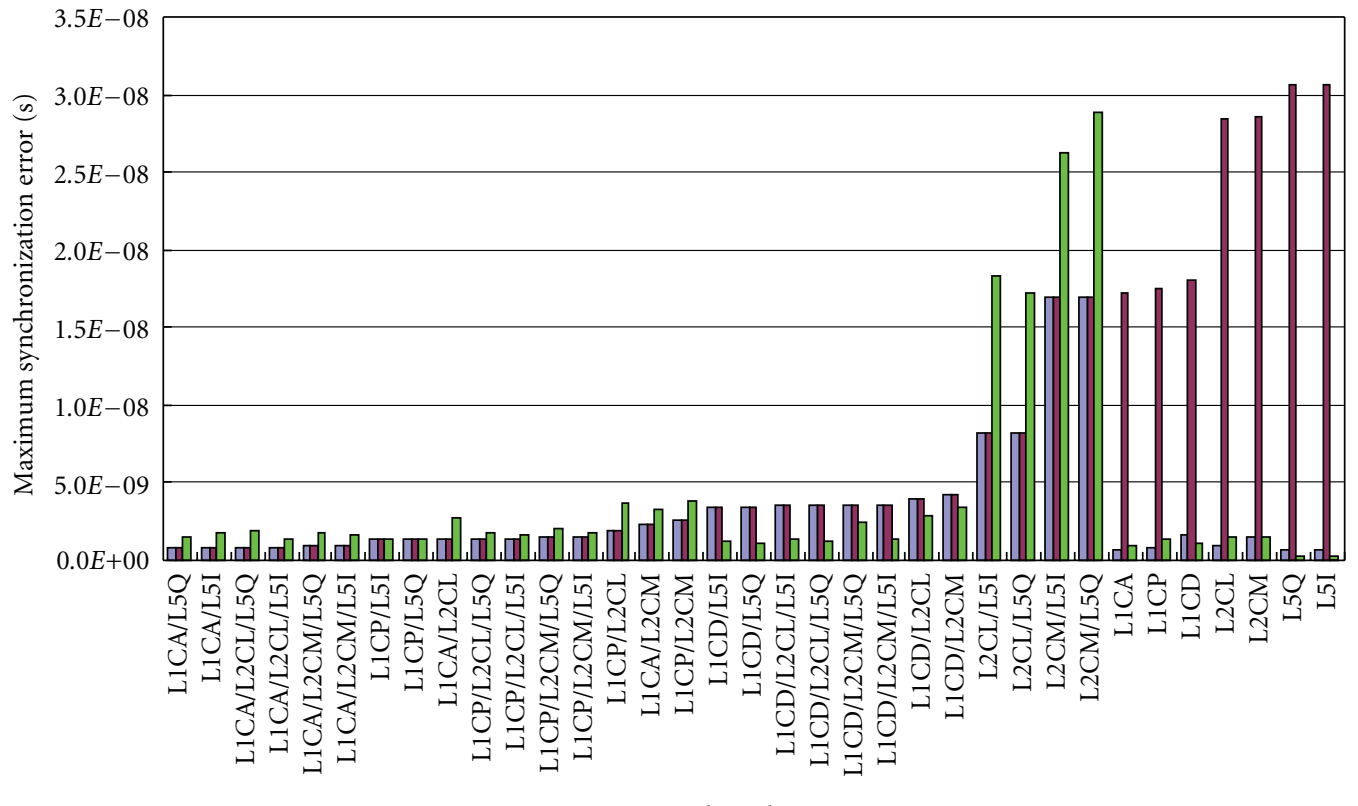

Signal combination

Case without ionospheric effect

Case with ionospheric effect

Experimental results

FIGURE 17: Estimated maximum synchronization error.

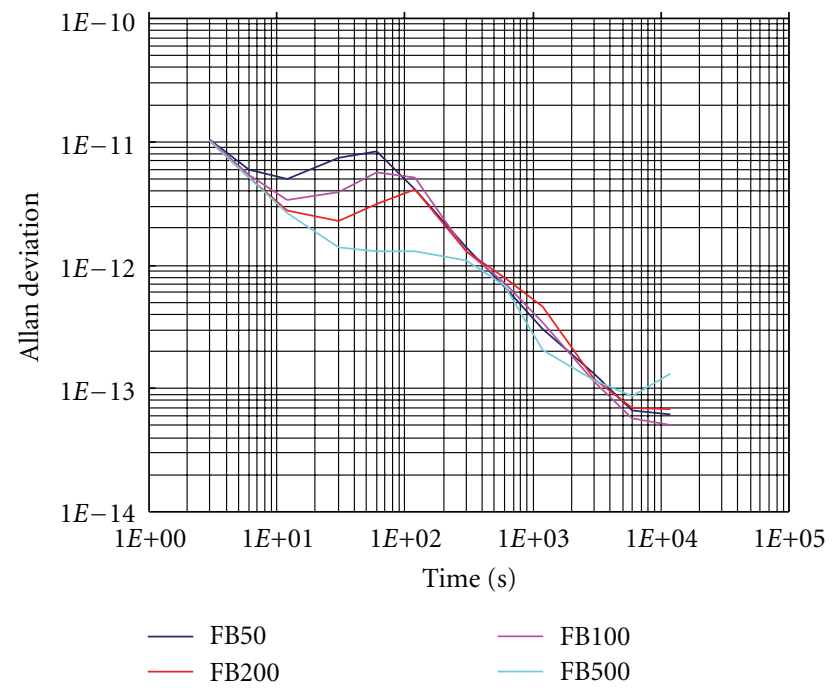

FIGURE 18: Allan deviations change with the number of values of time to be adjusted.

\section{Conclusions}

This study is summarized as follows:

(1) an error-adjustment method for RESSOX of the QZSS, where multiple frequency navigation signals are used, was demonstrated;

(2) feedback experiments of 35 combinations were conducted and some results were presented. Except for the L2/L5 combination, a synchronization error within $2 \mathrm{~ns}$ was achieved. Allan deviations in each experiment were also shown. The combination giving the best experimental results, excepting the singlefrequency signal, is the combination of L1CD and L5Q, and the expected maximum synchronization error is $1.14 \mathrm{~ns}$;

(3) a long-duration experiment was conducted with L1CA/L5I, and the overlapping Allan deviation was $1.23 \times 10^{-14}$ at $100,160 \mathrm{~s}$;

(4) The experimental results were compared with those of computer simulation, and the tendency of the experimental synchronization error was found to be the same as that of simulation. In the case of single-frequency, since it is difficult to estimate the ionospheric delay precisely, the synchronization error would be as large as those in the cases with ionospheric error of more than $10 \mathrm{~ns}$;

(5) one strategy for improving the stability of RESSOX control is to use 100 values of time to be adjusted (elapsed time for feedback control is $100 \mathrm{~s}$ ) in the convergence phase and 500 values (elapsed time for feedback control is $500 \mathrm{~s}$ ) in the steady phase in feedback control.

\section{Acknowledgments}

This study was carried out as part of the Basic Technology Development of Next-Generation Satellites project promoted by the Ministry of Economics, Trade and Industry 
(METI) through the Institute for Unmanned Space Experiment Free Flyer (USEF).

\section{References}

[1] M. Kishimoto, H. Hase, A. Matsumoto et al., "QZSS system design and its performance," in Proceedings of Institute of Navigation National Technical Meeting (NTM '07), pp. 405410, January 2007.

[2] J. J. Suter, L. J. Crawford, B. G. Montgomery, and W. E. Swann, "Syntonics LLC: APL-developed technology makes its commercial debut," Johns Hopkins APL Technical Digest, vol. 22, no. 2, pp. 168-175, 2001.

[3] T. Iwata, A. Iwasaki, Y. Fukuyama, T. Ikegami, H. Murakami, and K. Hagimoto, "Remote synchronization system for onboard crystal oscillator of quasi-zenith satellite system," in Proceedings of International Symposium on GPS/GNSS, pp. 375-380, Tokyo University of Marine Science and Technology, Tokyo, Japan, 2003.

[4] S. Kawase, "Satellite communication-navigation system with delay-error cancellation," IEICE Transactions on Communications (Japanese Edition), vol. J83-B, no. 10, pp. 1403-1409, 2000.

[5] Y. Asaki, H. Kobayashi, M. Ishiguro, H. Hirosawa, and H. Hirabayashi, "Development of the radiometric phase correction method using a 22-GHz water vapor line radiometer," IVS CRL-TDC News, no. 19, pp. 15-18, 2001.

[6] D. Giunta, G. Busca, A. Della Torre et al., "Recent developments in time \& frequency dissemination systems," in Proceedings on the 18th European Frequency and Time Forum, pp. 530-536, 2004.

[7] F. Tappero, A. Dempster, T. Iwata et al., "Proposal for a novel remote synchronization system for the on-board crystal oscillator of the quasi-zenith satellite system," Navigation, vol. 53, no. 4, pp. 219-229, 2006.

[8] T. Iwata, Y. Kawasaki, M. Imae et al., "Remote synchronization system of quasi-zenith satellites using multiple positioning signals for feedback control," Navigation, vol. 54, no. 2, pp. 99108, 2007.

[9] T. Iwata, M. Imae, T. Suzuyama et al., "Remote synchronization simulation of onboard crystal oscillator for QZSS using L1/L2/L5 signals for error adjustment," International Journal of Navigation and Observation, vol. 2008, Article ID 462062, 7 pages, 2008.

[10] T. Iwata, T. Matsuzawa, and Y. Hashibe, "Experimental apparatuses and tests of remote synchronization system of quasizenith satellites," in Proceedings of Institute of Navigation National Technical Meeting (NTM '08), pp. 477-486, January 2008. 

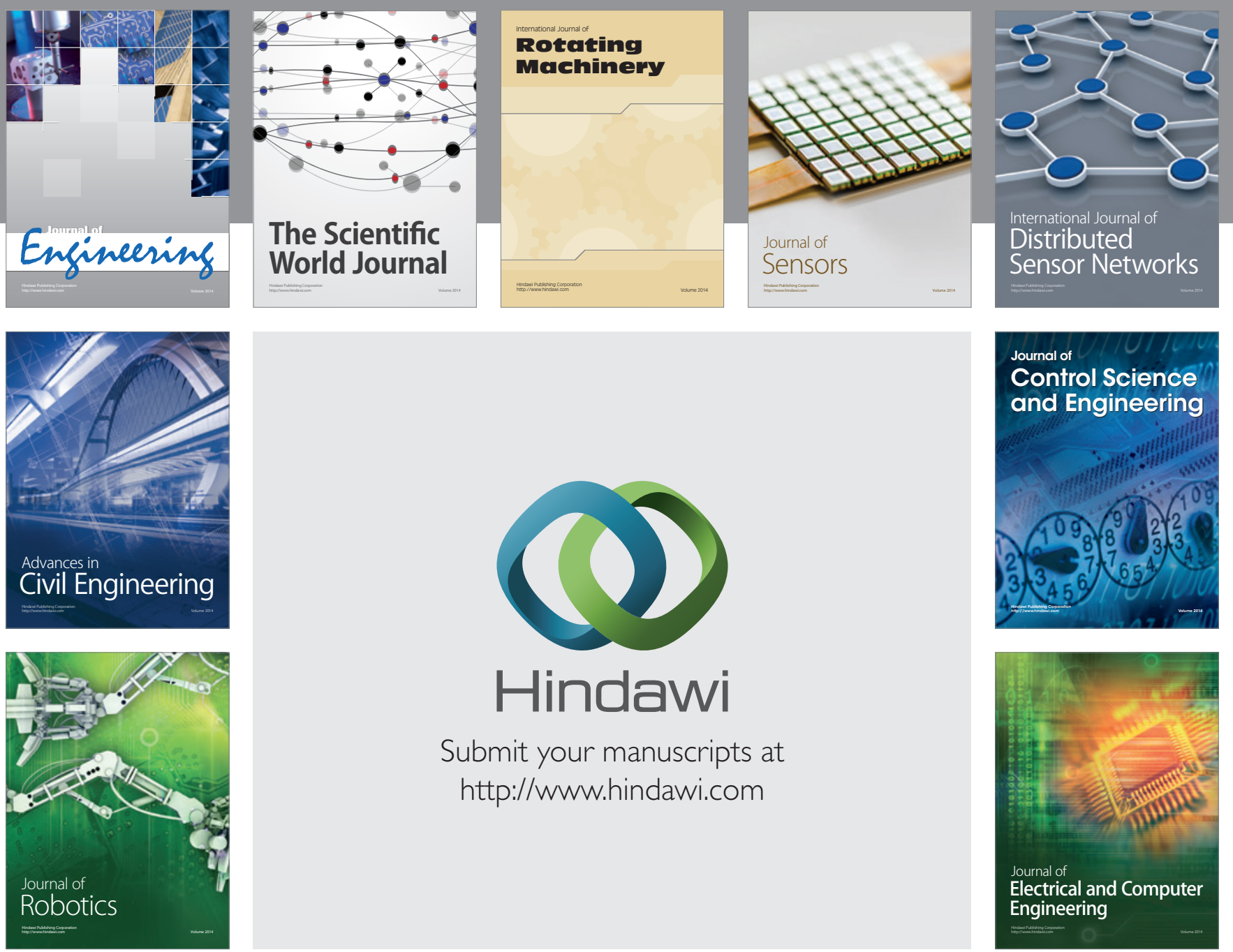

Submit your manuscripts at

http://www.hindawi.com
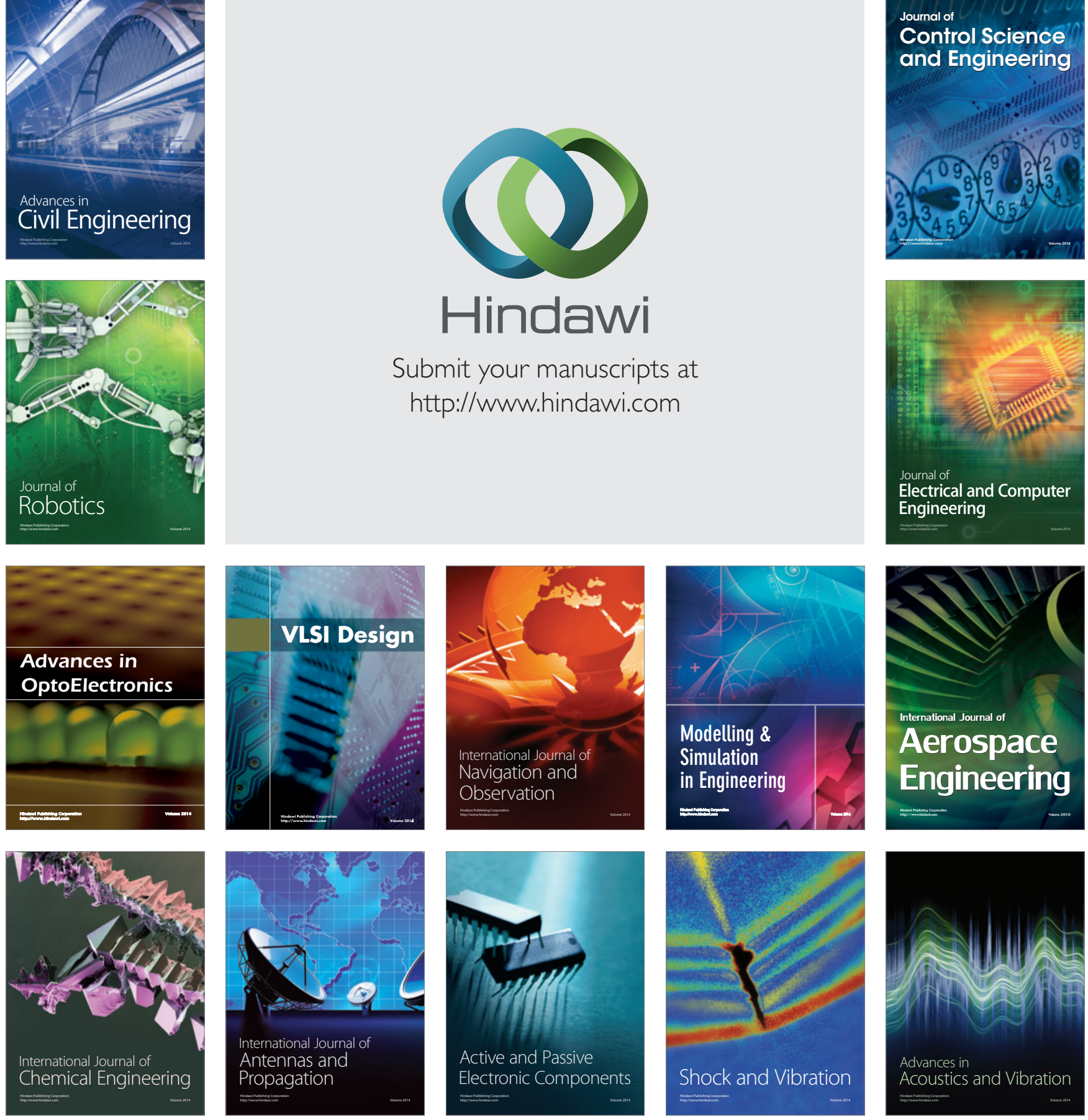